\title{
Osteopontin, E-cadherin, and $\beta$-catenin expression as prognostic biomarkers in patients with radically resected gastric cancer
}

\author{
Maria Di Bartolomeo ${ }^{1}$ - Filippo Pietrantonio ${ }^{1}$ Alessandro Pellegrinelli ${ }^{2} \cdot$ \\ Antonia Martinetti $^{1} \cdot$ Luigi Mariani $^{3} \cdot$ Maria Grazia Daidone $^{4} \cdot$ Emilio Bajetta $^{5}$. \\ Giuseppe Pelosi $^{2} \cdot$ Filippo de Braud $^{1} \cdot$ Irene Floriani $^{6} \cdot$ Rosalba Miceli $^{3}$
}

Received: 29 January 2015 / Accepted: 26 March 2015/Published online: 11 April 2015

(C) The International Gastric Cancer Association and The Japanese Gastric Cancer Association 2015

\begin{abstract}
A correlation between osteopontin, E-cadherin, $\beta$-catenin, and cyclooxygenase 2 overexpression and poor clinicopathological features and prognosis has been previously suggested in gastric cancer. This translational study was aimed at assessing the correlation of these immunohistochemical biomarkers with outcome in patients with radically resected gastric cancer. We analyzed osteopontin, E-cadherin, $\beta$-catenin, and cyclooxygenase 2 expression by immunohistochemistry in 346 primary gastric tumor tissue samples from patients enrolled in the ITACA-S trial. This phase III study randomized patients with radically resected gastric cancer to receive adjuvant chemotherapy with either 5 -fluorouracil and leucovorin or a sequential regimen of infusional 5-fluorouracil and leucovorin plus irinotecan followed by cisplatin and docetaxel. High expression of
\end{abstract}

Electronic supplementary material The online version of this article (doi:10.1007/s10120-015-0495-y) contains supplementary material, which is available to authorized users.

Maria Di Bartolomeo

maria.dibartolomeo@istitutotumori.mi.it

1 Medical Oncology Department, Fondazione IRCCS Istituto Nazionale dei Tumori, Via Venezian 1, 20133 Milan, Italy

2 Pathology Department, Fondazione IRCCS Istituto Nazionale dei Tumori, Milan, Italy

3 Unit of Medical Statistics, Biometry and Bioinformatics, Fondazione IRCCS Istituto Nazionale dei Tumori, Milan, Italy

4 Experimental Oncology and Molecular Medicine Department, Fondazione IRCCS Istituto Nazionale dei Tumori, Milan, Italy

5 Medical Oncology Unit, IDO Policlinico, Monza, Italy

6 Clinical Research Laboratory, IRCCS Istituto di Ricerche Farmacologiche "Mario Negri", Milan, Italy osteopontin was correlated with high histological grade, diffuse histotype, and peritoneal relapse, but not with TNM stage. Moreover, osteopontin overexpression was associated with higher risk of tumor recurrence and metastases, and was an independent prognostic factor for both relapsefree and overall survival of gastric cancer patients following adjuvant chemotherapy. Abnormal E-cadherin expression and abnormal $\beta$-catenin expression were correlated with more advanced disease stage, and as a consequence, with poor outcome. Our results suggest that osteopontin overexpression is a valuable independent predictor of tumor recurrence and survival in patients with radically resected gastric cancer.

Keywords Osteopontin · Gastric cancer - Prognosis · Biomarker · Adjuvant chemotherapy

\section{Introduction}

Gastric cancer (GC) is the second leading cause of cancerrelated death in the world [1]. Surgery is the mainstay of treatment for localized disease, but recurrence rates remain high even after radical resection [2]. The development of treatment strategies to prevent locoregional and systemic recurrences is a major challenge of current research. Thus, efforts to improve survival of patients with GC are mainly focused on the establishment of combined modality therapy consisting of appropriate surgery, perioperative or adjuvant chemotherapy, and radiotherapy [3-5].

The tumor-node-metastasis (TNM) staging system is used worldwide to drive therapeutic decisions according to prognostic stratification. However, GC is a heterogeneous disease with several epidemiological and histopathological characteristics, and with different molecular subtypes [6]. 
The molecular heterogeneity of GC is responsible for the considerable variability of patient outcomes-independently of disease stage - and highlights the unmet clinical need for validated prognostic biomarkers [7].

Alteration of the expression of adhesion molecules is a frequent event in GC [8]. In particular, E-cadherin plays an essential role in the maintenance of cell polarity and differentiation, in the contact inhibition of cell growth, and in the suppression of cancer invasion. Functional E-cadherin$\beta$-catenin adhesion complexes are involved in anchorage mechanisms, and $\beta$-catenin is also involved in the Wingless/Wnt signaling pathway and transcription activation. Osteopontin, an integrin-binding glycophosphoprotein cytokine, plays an important role in cancer cell adhesion, protection from apoptosis, invasion, and migration [9] via the degradation of extracellular matrix, angiogenesis, and cell survival [10]. Literature data have documented that osteopontin expression is related to metastatic potential, and may be a prognostic marker in several tumor types, including GC, and especially in patients with more advanced disease stage [9]. Moreover, osteopontin and cyclooxygenase 2 (COX-2), which is one of the rate-limiting enzymes for prostaglandin synthesis, could synergistically induce angiogenesis and metastasis in GC [11].

In the present study we analyzed the prognostic role of osteopontin, E-cadherin, $\beta$-catenin, and COX-2 in tumor tissue from patients with radically resected GC, with the aim of evaluating the association between their immunohistochemical expression pattern and patient outcomes.

\section{Materials and methods}

\section{Patient population and study design}

To conduct our investigations, we used data from the ITACA-S study [12], a multicenter randomized, open-label, parallel group, superiority phase III trial which planned to evaluate a survival benefit with sequential use of infusional 5-fluorouracil (5-FU) and leucovorin (LV) plus irinotecan (FOLFIRI regimen) followed by cisplatin and docetaxel versus 5-FU and LV alone (De Gramont regimen). The study population consisted of 1106 patients enrolled between 2005 and 2009 and with pathologically confirmed, radically resected, stage II/III adenocarcinoma involving the stomach or the gastroesophageal junction. The primary end point of disease-free survival, which may be considered as a surrogate for overall survival (OS) [13], was not significantly different among the two treatment arms [12].

In this ancillary study, a panel of immunohistochemical biomarkers-osteopontin, E-cadherin, $\beta$-catenin, and COX-2 - was determined in the primary gastric tumor tissues of the enrolled patients, with the aim of evaluating their association with disease characteristics and patient outcome in terms of relapse-free survival (RFS) and OS.

From the 104 centers participating to the ITACA-S study, we selected those contributing at least five relapsed patients. Of these 23 centers, 20 were able to collect biological samples, for a total of 346 patients with available tissue specimens. The protocol of the ancillary study was approved by the local institutional review boards of all participating centers and by the Italian Data Protection Authority. All living patients provided specific written informed consent.

Formalin-fixed, paraffin-embedded tumor tissue blocks were collected through the coordination of the Istituto di Ricerche Farmacologiche "Mario Negri" of Milan, which provided the database of clinicopathological characteristics and patient outcome data. Processing and analysis of tumor specimens was performed without knowledge of the patients' clinical data in the pathology department of the coordinating center, the Fondazione IRCCS Istituto Nazionale dei Tumori of Milan.

\section{Assessment of clinicopathological characteristics}

The 2009 TNM classification system was used for pathological staging [14]. Two senior gastrointestinal pathologists, both blinded to the other's evaluations and the patient outcomes, revised centrally all hematoxilin-eosin sections for diagnosis and tumor content and recorded their assessment of the pathological stage. In the case of disagreement, consensus between the pathologists was reached at the double-headed microscope. The primary tumor site was categorized as proximal if the bulk of the tumor (more than $80 \%$ ) was located in the gastric cardia with possible extension up to the gastroesophageal junction and a small portion of the distal esophagus, or as distal in the mid body of the stomach or down to the pylorus in the other cases. Tumor histotype was classified in two categories according to the Lauren classification: diffuse-type GC or intestinaltype GC. Rare histotypes were excluded. Tumor histological grade was categorized as grade 1 or grade 2 if the tumor was well or moderately differentiated and grade 3 if it was poorly differentiated or undifferentiated.

\section{Immunohistochemical analysis and evaluation}

Immunohistochemical staining for osteopontin, E-cadherin, $\beta$-catenin, and COX-2 was done using an EnVision ${ }^{\circledR}$ FLEX+ detection system. Formalin-fixed, paraffin-embedded tissue blocks were sectioned $(3 \mu \mathrm{m})$, placed on 3-aminopropyltriethoxysilane-coated slides and anonymized by a coding system. After deparaffinization, endogenous peroxidise activity was blocked by $\mathrm{H}_{2} \mathrm{O}_{2}$. The primary antibody for osteopontin was a mouse monoclonal 
antibody (Novus Biologicals clone 1b20) used at dilution of 1:800. Antigen retrieval was performed by heating in our $10 \mathrm{mM}$ citrate buffer in PT Link (Dako ${ }^{\circledR}$ ). Negative controls were obtained by omission of the primary antibodies, and normal gastric oxyntic glands were used as positive controls. For E-cadherin and $\beta$-catenin, the primary antibody was a mouse monoclonal antibody (BD Transduction Laboratories $^{\text {TM}}$; clones 36 and 14, used at dilutions 1:2000 and 1:1000, respectively).

The results were read by the two qualified pathologists without any prior knowledge of each patient's clinical information. When the opinions of the two evaluators were different, consensus was reached by discussion. The degree of cytoplasmatic immunoreactivity for osteopontin was evaluated according to the modified method proposed by Ito et al. [15]. Staining was evaluated in ten fields per section at $\times 200$ magnification of two slides from each specimen to classify samples into three groups according to the osteopontin expression intensity: $0 / 1+$ for negative or weakly positive expression; $2+$ for moderate expression or focal (less than $50 \%$ of tumor) strongly positive expression; and $3+$ for extended ( $50 \%$ or more of the tumor) strongly positive expression (Fig. 1). For E-cadherin and $\beta$-catenin, staining intensity was graded semiquantitatively from 0 to 3 , as previously described [16]: 0 for negative staining; 1 for cytoplasmic staining; 2 for heterogeneous staining (tumors comprising both normal and abnormal staining areas); and 3 for a normal membranous staining. Because the staining pattern sometimes differed within the same tumor, particularly when the differentiation status differed, the final score was based on the dominant pattern. For data analysis, all tumors with loss of membranous expression were classified as abnormal and included those with absent, heterogeneous, or cytoplasmic staining patterns. For COX-2, extended (50\% or more of the tumor) strongly positive staining was considered as overexpression.

\section{Statistical analysis}

Binary associations between categorical variables were tested with the exact chi square test.

The main study variables were osteopontin expression $(0 / 1+, 2+$, or $3+)$, E-cadherin (normal, abnormal), $\beta$ catenin (normal, abnormal), and COX-2 (normal, overexpressed). The end points were RFS and OS. The RFS time was calculated from the randomization date to the date of disease relapse or death without evidence of disease, whichever occurred first; the RFS time was equal to the censoring time for relapse-free patients. The OS time was calculated from the randomization date to the date of death, regardless of the cause, or as equal to the censoring time for living patients. RFS and OS curves were estimated by the Kaplan-Meier method and were compared with the log-rank test. Multivariate analyses of RFS and OS were performed using Cox models; together with the study variables, the models included pathological TNM stage (IB/IIA, IIB/IIIA, IIIB, IIIC), histological grade (G1/G2, G3), and histotype (intestinal type, diffuse type) as adjustment factors. Following some literature reports [8], additional multivariate Cox model analyses were performed aimed at investigating whether the osteopontin prognostic effect was different according to the tumor stage by including in the models the interaction between osteopontin and tumor stage (IB/IIIA, IIIB/IIIC), together with the other biomarkers and the adjustment factors.

The analyses were performed using $\mathrm{SAS}^{\circledR}$ and R software. We considered a statistical test result as significant when the corresponding two-sided $p$ value was less than $5 \%$. In the models, the missing values ( 1 for E-cadherin, 2 for $\beta$-catenin, and 1 for COX-2) were replaced by the modal category; in all the other analyses they were excluded.

\section{Results}

\section{Patients characteristics}

The demographics and disease characteristics of the 346 patients analyzed are summarized in Table 1. According to the 2009 TNM classification system, approximately $51 \%$ of tumors was classified as pathological stage IIIB/IIIC. Regarding tumor histotype, 167 samples (48\%) were thought to be of diffuse type, and 179 samples (52\%) were thought to be of intestinal type. Histological features were well to moderately differentiated (G1/G2) in 103 patients (30\%) and poorly differentiated (G3) in 243 patients (70\%). The median number of lymph nodes retrieved was 26 (interquartile range, IQR, 19-36), and the median number of positive nodes was 5 (IQR, 2-12).

None of the patients were lost to follow-up. The median follow-up was 61 months (IQR, 48-75 months). One hundred forty-eight patients relapsed and died of the disease, whereas 19 patients who relapsed were alive at the last follow-up and 31 died without any previous relapse; the overall number of deaths was 179. Disease outcome was similar to that reported in the ITACA-S study [11]: the 6-year RFS and OS rates were $39.9 \%$ (95\% confidence interval, CI, 34.4-46.3\%; median RFS, 38 months) and $46.9 \%$ (95\% CI, 41.6-52.9\%; median OS, 55 months), respectively.

\section{Immunohistochemical biomarkers and their association with other clinicopathological variables}

The complete results for the association between the immunohistochemical biomarkers and other clinicopathological 


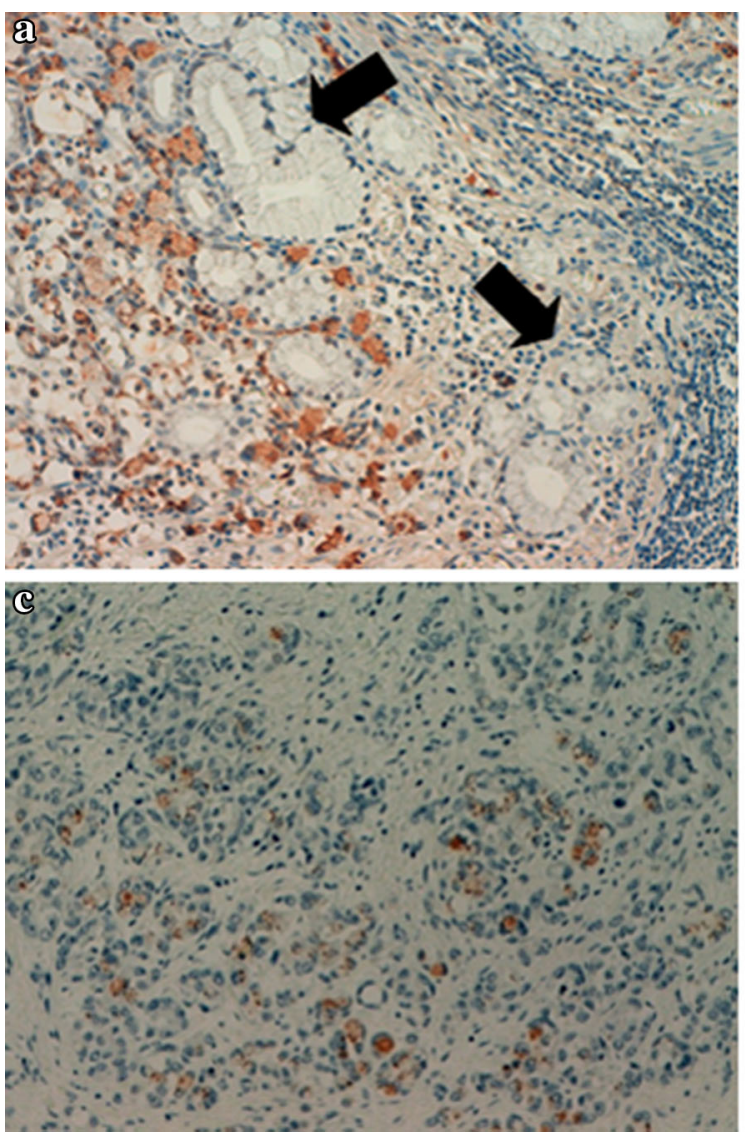

Fig. 1 Examples of immunohistochemical expression of osteopontin. a Physiological absence of immunoreactivity for osteopontin in gastric foveolar epithelium (negative control); tumor cells show $3+$ osteopontin expression. b Nearly total absence of immunoreactivity for osteopontin in a sample of poorly differentiated gastric cancer

variables are shown in Table S1. Regarding osteopontin expression, $188(54 \%)$ of the primary gastric tumors had a osteopontin expression score of $0 / 1+, 82(24 \%)$ had a score of $2+$, and $76(22 \%)$ had a score of $3+$. E-cadherin showed normal membrane pattern in 241 samples $(70 \%)$ and abnormal expression in 104 samples (30\%). $\beta$-Catenin showed normal membrane pattern in 155 samples $(45 \%)$ and abnormal expression in 189 samples (55\%). COX-2 was classified as overexpressed in only 27 cases $(8 \%)$ (Table 1$)$. Osteopontin expression was significantly associated with histotype $(p=0.001)$; in particular, the percentage of $3+$ tumors was greater in the diffuse type than in the intestinal type (30\% vs $15 \%$ ), whereas the percentage of negative tumors was similar in the two histotype groups (53\% vs $56 \%$, respectively). Similarly, osteopontin expression was significantly associated with higher histological grade $(p=0.044)$; poorly differentiated tumors showed a greater percentage of $3+$ osteopontin expression as compared with well or moderately differentiated tumors ( $26 \%$ vs $14 \%$ ). As regards pathological TNM stage, higher osteopontin score
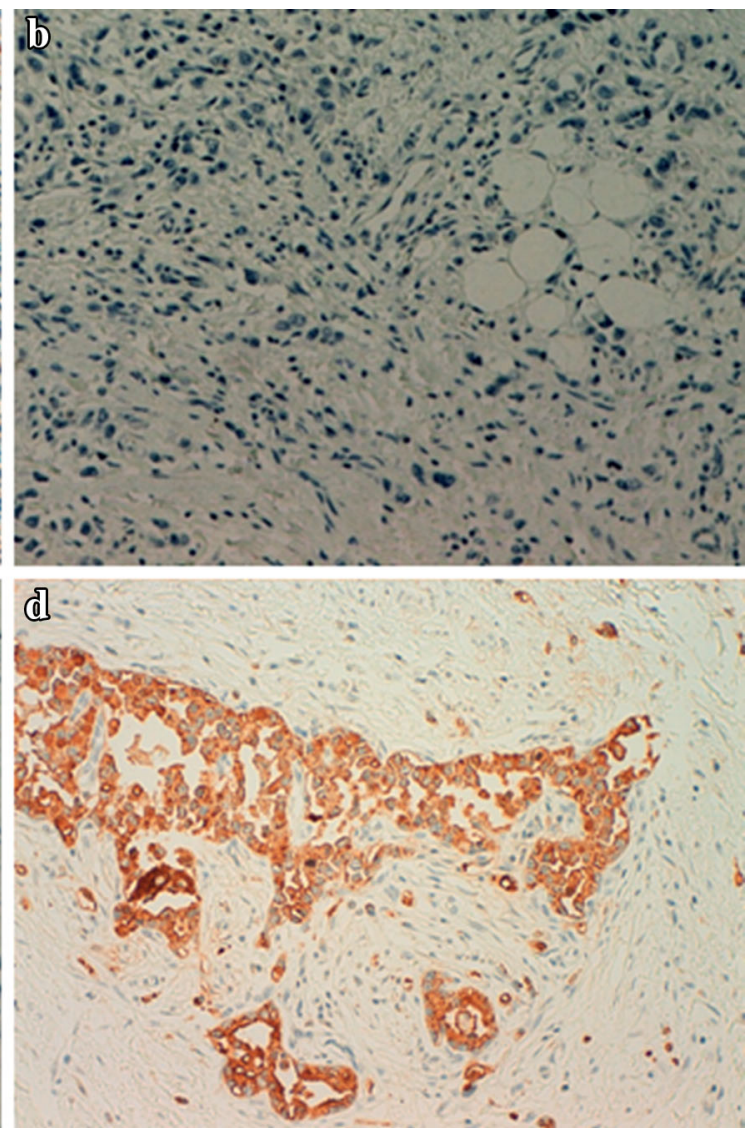

(score 0/1+). c Focal immunoreacitivity (less than $50 \%$ ) for ostepontin in a sample of moderately differentiated gastric cancer (score 2+). d Diffuse immunoreactivity (more than 50\%) for osteopontin in a sample of poorly differentiated gastric cancer (score $3+)$

was not significantly correlated with more advanced disease $(p=0.131)$. We only observed a slightly greater percentage of tumors with $3+$ osteopontin expression in patients with stage IIIB/IIIC disease as compared with the other patients (26\% vs $18 \%$ ). The two treatment arms were well balanced as regards the proportion of tumors with high (3+) osteopontin expression ( $22 \%$ in the $5-\mathrm{FU}$ and $\mathrm{LV}$ arm and $22 \%$ in the sequential treatment arm; $p=0.141$ ). A clinically meaningful difference was observed in terms of peritoneal relapse in patients with $3+$ osteopontin expression $(43 \%)$ versus $0 / 1+$ and $2+$ osteopontin expression (18 and $16 \%$, respectively).

No significant associations were highlighted between the primary tumor site and the biomarkers (Table S1). Finally, abnormal $\beta$-catenin expression and abnormal E-cadherin expression were correlated with each other $(p<0.001)$, and with diffuse histotype or poorly differentiated cancer (all $p<0.001)$. Importantly, these biomarkers were also correlated with more advanced disease stage, i.e., IIIB/IIIC ( $p=0.005$ and $p<0.001$, respectively). 
Table 1 Patients and disease characteristics

\begin{tabular}{|c|c|}
\hline & Value $^{\mathrm{a}}$ \\
\hline \multicolumn{2}{|l|}{ Age (years) } \\
\hline Median & 63 \\
\hline Interquartile range & $55-68$ \\
\hline \multicolumn{2}{|l|}{ Gender } \\
\hline Male & $226(65 \%)$ \\
\hline Female & $120(35 \%)$ \\
\hline \multicolumn{2}{|l|}{ Treatment arm } \\
\hline 5-FU and LV & $171(49 \%)$ \\
\hline FOLFIRI followed by CDDP and TXT & $175(51 \%)$ \\
\hline \multicolumn{2}{|l|}{ Status of the primary tumor infiltration } \\
\hline $\mathrm{T} 1$ & $25(7 \%)$ \\
\hline $\mathrm{T} 2$ & $51(15 \%)$ \\
\hline $\mathrm{T} 3$ & $101(29 \%)$ \\
\hline $\mathrm{T} 4$ & $169(49 \%)$ \\
\hline \multicolumn{2}{|l|}{ Status of the primary tumor nodes } \\
\hline No & $26(8 \%)$ \\
\hline N1 & $82(24 \%)$ \\
\hline $\mathrm{N} 2$ & $83(24 \%)$ \\
\hline $\mathrm{N} 3 \mathrm{a}$ & $96(28 \%)$ \\
\hline $\mathrm{N} 3 \mathrm{~b}$ & $59(17 \%)$ \\
\hline \multicolumn{2}{|l|}{ Pathological TNM stage } \\
\hline IB & $14(4 \%)$ \\
\hline IIA & $34(10 \%)$ \\
\hline IIB & $67(19 \%)$ \\
\hline IIIA & $56(16 \%)$ \\
\hline IIIB & $76(22 \%)$ \\
\hline IIIC & $99(19 \%)$ \\
\hline \multicolumn{2}{|l|}{ Histotype } \\
\hline Intestinal & $179(52 \%)$ \\
\hline Diffuse & $167(48 \%)$ \\
\hline \multicolumn{2}{|l|}{ Histological grade } \\
\hline G1/G2 & $103(30 \%)$ \\
\hline G3 & $243(70 \%)$ \\
\hline \multicolumn{2}{|l|}{ Primary tumor site } \\
\hline Proximal & $63(18 \%)$ \\
\hline Distal & $283(82 \%)$ \\
\hline \multicolumn{2}{|l|}{ Osteopontin expression } \\
\hline $0 / 1+$ & $188(54 \%)$ \\
\hline $2+$ & $82(24 \%)$ \\
\hline $3+$ & $76(22 \%)$ \\
\hline \multicolumn{2}{|l|}{ E-cadherin expression ${ }^{a}$} \\
\hline Normal & $241(70 \%)$ \\
\hline Abnormal & $104(30 \%)$ \\
\hline \multicolumn{2}{|l|}{$\beta$-Catenin expression ${ }^{\mathrm{b}}$} \\
\hline Normal & $155(45 \%)$ \\
\hline Abnormal & $189(55 \%)$ \\
\hline COX-2 expression ${ }^{\mathrm{a}}$ & \\
\hline
\end{tabular}

Table 1 continued

\begin{tabular}{ll}
\hline & Value $^{\mathrm{a}}$ \\
\hline Normal & $318(92 \%)$ \\
Overexpression & $27(8 \%)$ \\
\hline The values given are the number and the percentage (in parentheses), \\
except for age. \\
CDDP cisplatin, COX-2 cyclooxygenase 2, FOLFIRI infusional \\
5-fluorouracil and leucovorin plus irinotecan, 5-FU 5-fluorouracil, LV \\
leucovorin, TNM tumor-node-metastasis, TXT docetaxel \\
${ }^{a}$ One missing value excluded \\
${ }^{\mathrm{b}}$ Two missing values excluded
\end{tabular}

Patient outcome according to osteopontin, E-cadherin, $\beta$-catenin, and COX-2 expression

Univariate Kaplan-Meier analysis showed that osteopontin expression was significantly associated with RFS and OS ( $p<0.001$ and $p<0.002$, respectively) (Fig. 2a, b). The 6-year RFS rate was $49.7 \%$ (95\% CI, 42.7-57.9\%) in the $0 / 1+$ osteopontin expression group versus $34.0 \%$ (95\% CI, 23.3-49.5\%) in the $2+$ osteopontin expression group versus $22.9 \%$ (95\% CI, 14.1-37.1\%) in the 3+ osteopontin expression group. The 6-year OS rate was $53.0 \%$ (95\% CI, 45.8-61.4\%) in the 0/1+ osteopontin expression group versus $43.2 \%$ (95\% CI, 32.7-57.2\%) in the $2+$ osteopontin expression group versus $34.2 \%(95 \% \mathrm{CI}$, $24.7-47.5 \%)$ in the $3+$ osteopontin expression group. E-cadherin expression was significantly associated with RFS and OS ( $p=0.003$ and $p=0.001$, respectively). The 6-year RFS rate was $47.1 \%$ (95\% CI, 40.9-54.1\%) for normal expression versus $22.8 \%$ (95\% CI, 14.1-37.2\%) for abnormal expression. The 6-year OS rate was $51.0 \%$ (95\% CI, 44.5-58.4\%) versus $37.5 \% \quad(95 \%$ CI, 29.0-48.3\%), respectively.

The expression of $\beta$-catenin showed a trend for association with RFS and a significant correlation with OS ( $p=0.054$ and $p=0.009$, respectively). The 6-year RFS rate was $46.3 \%$ (95\% CI, 38.8-55.3\%) for normal expression versus $33.9 \%$ (95\% CI, 26.6-43.3\%) for abnormal one. The 6-year OS rate was $52.0 \%(95 \% \mathrm{CI}$, $44.2-61.2 \%$ ) versus $42.4 \%$ (95\% CI, 35.5-50.7\%), respectively. COX-2 was not significantly associated with both outcomes (data not shown).

On multivariate Cox model analysis, among the four immunohistochemical biomarkers, only osteopontin showed a significant association with both RFS and OS (Table 2; $p=0.001$ and $p=0.017$, respectively). For the adjustment factors, TNM stage was highly significant as expected (both $p<0.001$ ) and histotype was significant for RFS only $(p=0.026)$. 

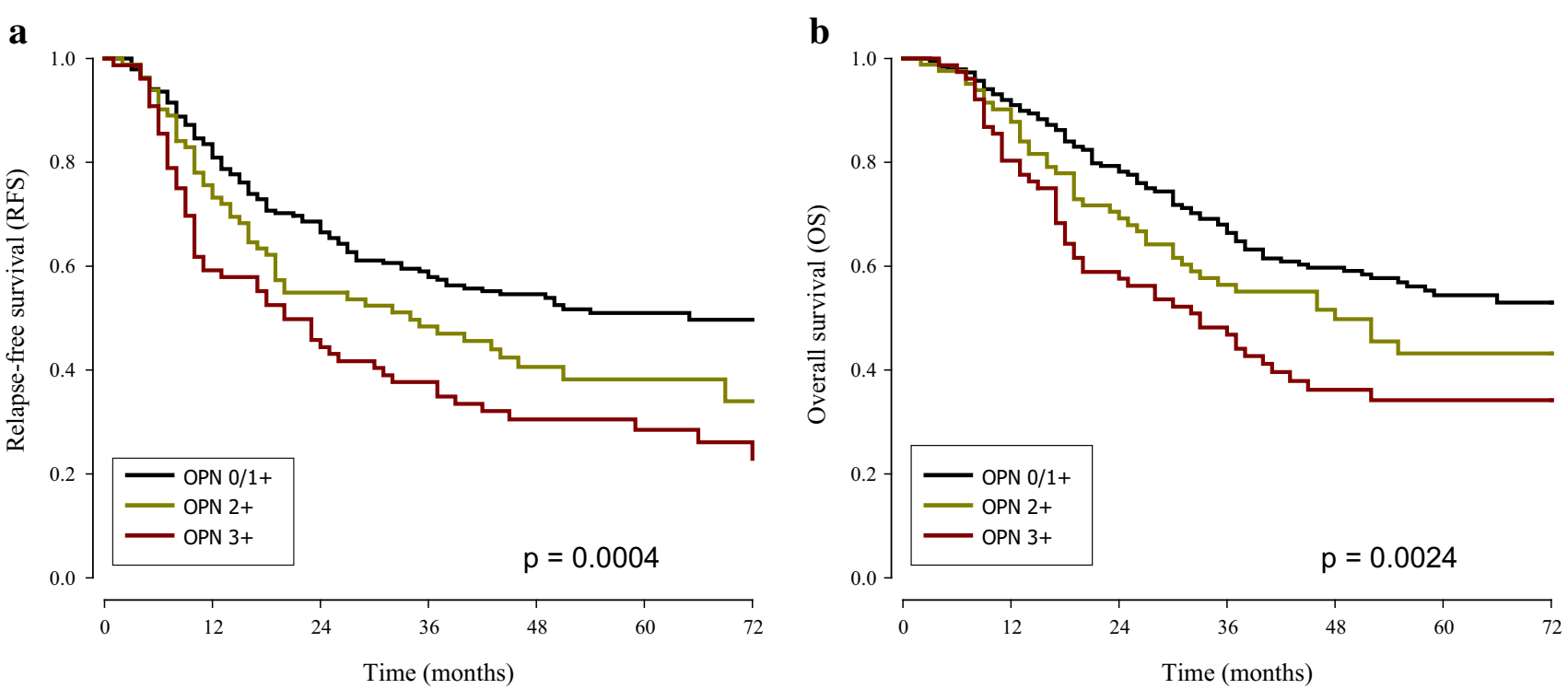

Fig. 2 Kaplan-Meier curves for relapse-free survival (a) and overall survival (b) according to the osteopontin $(O P N)$ expression groups: 0/1+ (188 patients) versus $2+$ (82 patients) versus $3+$ (76 patients)

Table 2 Multivariate Cox model analyses of relapse-free survival (RFS) and overall survival $(O S)$

\begin{tabular}{|c|c|c|c|c|c|c|}
\hline & \multicolumn{3}{|l|}{ RFS } & \multicolumn{3}{|l|}{ OS } \\
\hline & HR & $\mathrm{CI}$ & $p$ & HR & CI & $p$ \\
\hline \multicolumn{7}{|l|}{ Pathological TNM stage } \\
\hline IIB/IIIA vs IB/IIA & 2.67 & $1.26-5.63$ & $<0.0001$ & 2.95 & $1.26-6.95$ & $<0.0001$ \\
\hline IIIB vs IB/IIA & 5.26 & $2.48-11.14$ & & 6.01 & $2.55-14.13$ & \\
\hline IIIC vs IB/IIA & 11.57 & $5.54-24.13$ & & 12.51 & $5.42-28.85$ & \\
\hline Histological grade: G3 vs G1/G2 & 1.38 & $0.92-2.06$ & 0.121 & 1.46 & $0.95-2.23$ & 0.084 \\
\hline Histotype: diffuse vs intestinal & 1.51 & $1.05-2.17$ & 0.026 & 1.41 & $0.97-2.04$ & 0.074 \\
\hline \multicolumn{7}{|l|}{ Osteopontin expression } \\
\hline $2+$ vs $0 / 1+$ & 1.45 & $1.02-2.06$ & 0.001 & 1.40 & $0.97-2.04$ & 0.017 \\
\hline $3+$ vs $0 / 1+$ & 1.85 & $1.32-2.61$ & & 1.64 & $1.15-2.34$ & \\
\hline E-cadherin expression: abnormal vs normal & 1.13 & $0.80-1.61$ & 0.481 & 1.14 & $0.79-1.65$ & 0.491 \\
\hline$\beta$-Catenin expression: abnormal vs normal & 1.02 & $0.72-1.45$ & 0.908 & 1.11 & $0.76-1.62$ & 0.586 \\
\hline COX-2 expression: overexpressed vs normal & 1.13 & $0.69-1.87$ & 0.619 & 0.97 & $0.56-1.66$ & 0.898 \\
\hline
\end{tabular}

The $p$ value is from the two-sided Wald test

CI $95 \%$ confidence interval of hazard ratio, COX-2 cyclooxygenase 2, HR hazard ratio, TNM tumor-node-metastasis

\section{Subgroup analyses}

Available literature data suggested that osteopontin expression acts as a biomarker of clinical aggressiveness in patients with more advanced disease stage [9]. In our study, in patients with earlier stages of disease (IB/IIIA), 3+ osteopontin expression was not significantly associated with patient outcome $(p=0.476$ for RFS and $p=0.774$ for OS; curves not shown). On the other hand, in patients with stage IIIB/IIIC disease, the 6-year RFS rate was $30.0 \%$ (CI, 21.6-41.6\%) in the $0 / 1+$ osteopontin expression group versus $8.4 \%(\mathrm{CI}, 1.8-40.5 \%)$ in the $2+$ osteopontin expression group versus $4.3 \%$ (CI, $0.8-23.2 \%$ ) in the $3+$ osteopontin expression group $(p<0.001$; Fig. 3a). A similar result was observed for OS, with a statistically significant effect on OS observed only for stage IIIB/IIIC disease: the 6-year OS rate was $32.4 \%$ (CI, 23.2-45.2\%) in the $0 / 1+$ osteopontin expression group versus $12.8 \%$ (CI, 4.1-39.7\%) in the 2+ osteopontin expression group versus $11.4 \%(\mathrm{CI}, 5.0-26.9 \%)$ in the $3+$ osteopontin expression group ( $p=0.001$; Fig. 3b). However, multivariate analysis showed the osteopontin prognostic effect 

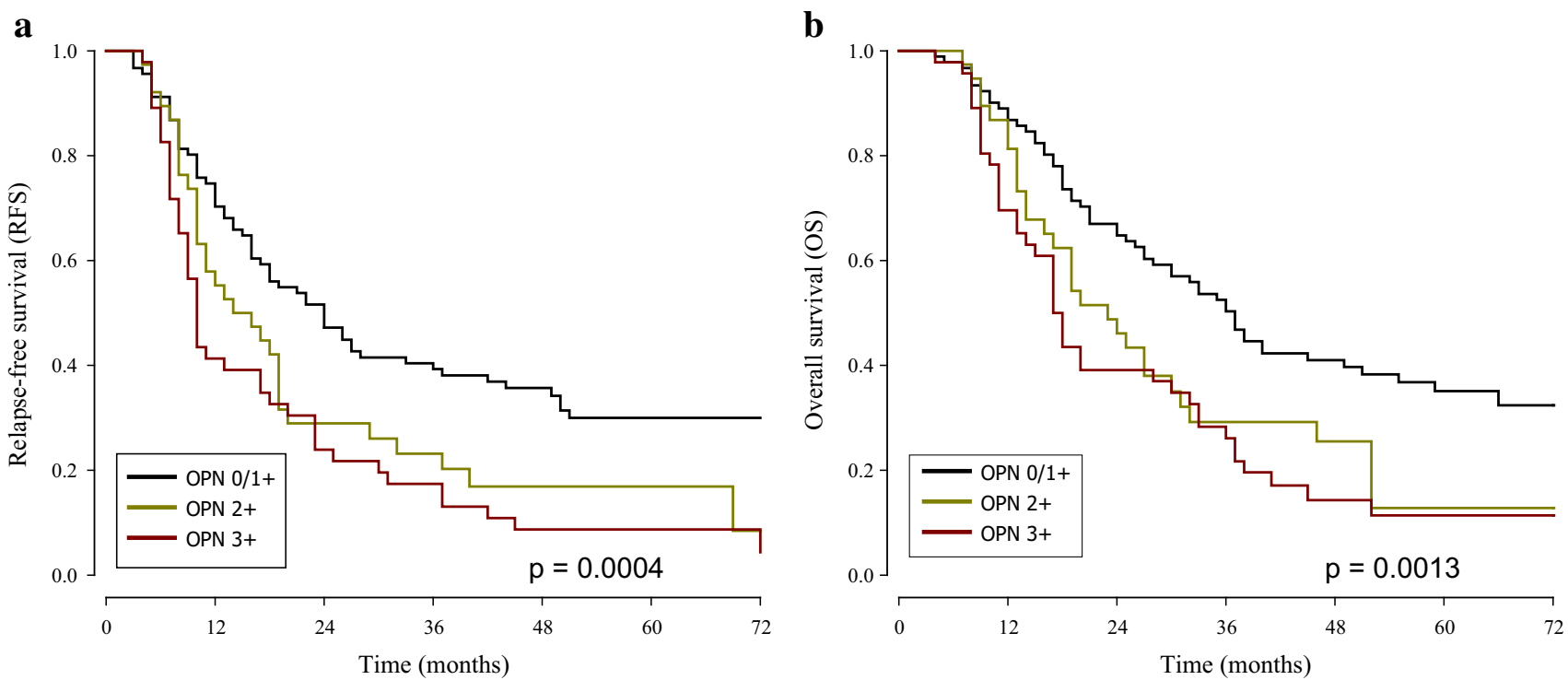

Fig. 3 Kaplan-Meier curves for relapse-free survival (a) and overall survival (b) according to the osteopontin $(O P N)$ expression groups: 0/1+ (91 patients) versus $2+$ (38 patients) versus $3+$ (46 patients) in the stage IIIB/IIIC disease subgroup

was not significantly different according to the tumor stage ( $p$ for interaction between osteopontin and the tumor stage was 0.557 for RFS and 0.456 for OS).

We finally speculated that the benefit of sequential treatment (FOLFIRI followed by cisplatin and docetaxel) could be different according to osteopontin expression. In the $0 / 1+$ osteopontin expression group, no major differences were observed in the RFS and OS curves $(p=0.882)$, whereas in the $3+$ osteopontin expression group, the 6-year RFS rate was $12.4 \%$ (CI, 4.8-31.8\%) in the 5-FU and $\mathrm{LV}$ arm versus $36.7 \%$ (CI, 23.4-57.4\%) in the sequential polychemotherapy $\operatorname{arm}(p=0.075)$; in the $2+$ osteopontin expression group the difference was less marked $(p=0.301)$. A similar trend was observed for OS $(p=0.598,0.164$ and 0.121 for the three osteopontin groups, respectively); in the $3+$ osteopontin expression group, the 6-year estimates were $22.8 \%$ (CI, $12.1-43.1 \%$ ) and $45.0 \%$ (CI, 31.1-65.1\%), respectively. Figure 4 shows RFS and OS curves according to the treatment arm in the group of patients with $3+$ osteopontin expression.

\section{Discussion}

Currently, only clinical prognostic factors are available to drive the treatment decision-making in patients with radically resected GC [17]. Pathological TNM stage is routinely used to assess prognosis and predict the need for adjuvant treatment. Since GC is a heterogeneous disease, tissue biomarkers are a promising tool for stratifying patient outcomes according to tumor biological characteristics, to be possibly integrated within available prognostic tools $[18,19]$. Active research is obtaining insight into the molecular characterization of the various genetic pathways involved in GC.

In our study, RFS and OS were significantly worse for patients with osteopontin overexpression and also for those with abnormal E-cadherin and $\beta$-catenin expression (Fig. 2), whereas E-cadherin and $\beta$-catenin were not independent prognostic factors.

These findings are of interest in view of the possible improvement in prognostic assessment through the combination of clinical and biological information. Moreover, our results are strengthened by the evidence of a weak association between disease stage and osteopontin expression, or its differential prognostic effect (i.e., the prognostic impact of osteopontin was not stage dependent). We found that $3+$ osteopontin expression was significantly associated with poorly differentiated and diffuse-type GC, whereas we excluded a significant association between osteopontin expression and pathological stage. This observation was in contrast with the results for abnormal E-cadherin and $\beta$ catenin expression, which were stage dependent and, consequently, not significantly associated with outcomes on multivariate analysis.

Osteopontin may stimulate several pathological processes in GC—-such as chronic inflammation, angiogenesis, cell survival, and tumor invasion and metastases-and may be associated with unfavorable clinicopathological features, including high histological grade, lymphovascular invasion, deep invasion of the gastric wall, and distant metastases [20-22]. A significant correlation between osteopontin overexpression and intestinal-type GC emerged 


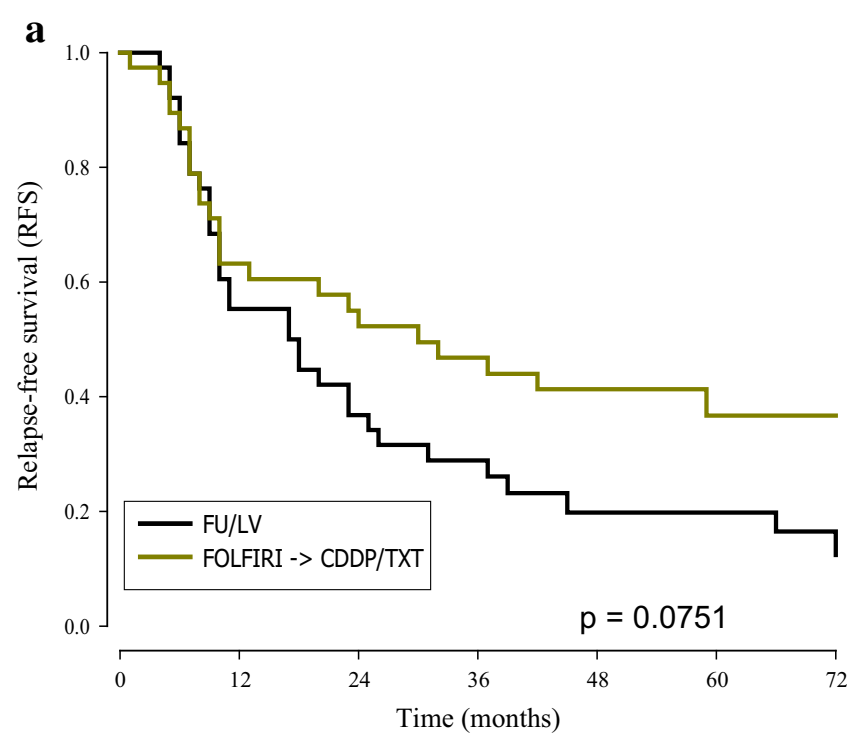

Fig. 4 Kaplan-Meier curves for relapse-free survival (a) and overall survival (b) according to treatment arm: 5-fluorouracil (5-FU) and leucovorin $(L V)$ (38 patients) versus 5-FU and LV plus irinotecan

from a small series [22], but was excluded by larger data sets $[20,21]$.

The functioning of the osteopontin network may be measured through several factors, such as plasma values, tissue messenger RNA levels or protein expression, and promoter polymorphisms [20-24].

It was previously reported that higher osteopontin plasma levels were independently associated with worse patient outcome [23]. However, the plasma concentration of osteopontin may be influenced by several nonneoplastic physiopathological processes and also by the complex tumor-microenvironment-related interactions. In our study, the immunohistochemical analysis offered the potential advantages of a less expensive and easy-to-perform technique-with well-known limitations constituted by the low reproducibility and interobserver variability. Therefore, in the present study, two blinded pathologists performed the centralized review of tumor histological grade and osteopontin scores.

Previous retrospective series evaluated osteopontin immunohistochemical expression as predictor of prognosis in patients treated with surgery with discordant results [2022]. Higashiyama et al. [21] explored the prognostic role of osteopontin expression in the largest ever reported retrospective series of 295 patients with curatively resected GC-failing to show a significant association on multivariate analysis.

The peculiarity of the present study consists in analyzing the prognostic and predictive impact of biologically relevant biomolecular features against robust traditional clinicopathological factors within the context of a clinical

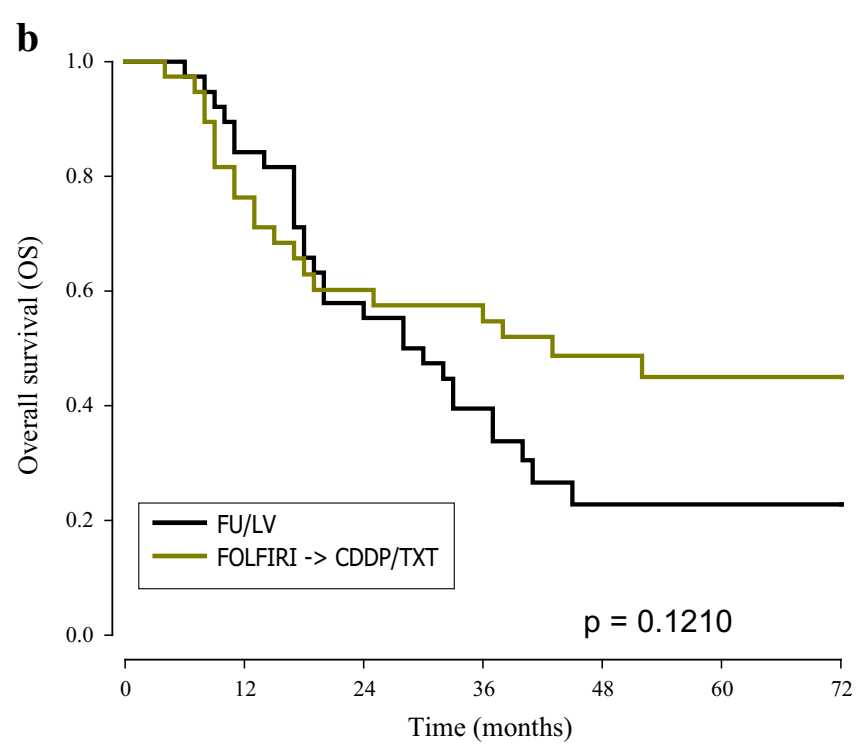

(FOLFIRI) followed by cisplatin (CDDP) and docetaxel (TXT) (38 patients) in the $3+$ osteopontin expression group

protocol, in which patients are homogeneous for tumor pathological stage and treatment. For this reason our data strongly support the use of osteopontin as a new biomarker that may help to improve outcome prediction and to drive treatment decision-making in patients undergoing resection of GC. Moreover, targeting osteopontin and its related signaling pathway-with monoclonal antibodies or small interfering RNAs - may constitute a promising strategy in the future treatment of patients [25]. Our study is the first step toward the discovery of prognostic and predictive biomarkers and the personalization of treatments through newer agents targeting the molecular drivers of the neoplastic progression [26].

Finally, our data showed that the poorer outcome associated with osteopontin overexpression was maintained only in the subset of patients with more advanced disease stage, i.e., IIIB/IIIC (Fig. 3). In fact, osteopontin may drive a particularly aggressive phenotype that is acquired only in the presence of other permissive factors emerging at later stages of GC progression. Similarly, in patients with poorer prognosis-i.e., those with $3+$ osteopontin expression, sequential polychemotherapy was associated with a trend for survival benefit compared with 5-FU alone (Fig. 4). However, all these observations derive from nonprespecified subgroup analyses and, although intriguing, should be considered carefully as hypothesis-generating. In particular, intensification of adjuvant treatment could be investigated prospectively in the high-risk subgroup of patients with more advanced disease stage and osteopontin overexpression.

In conclusion, we provided new and promising data on the association of osteopontin expression and poor outcome 
in patients with radically resected GC. Further prospective biomarker-driven studies are warranted to confirm the clinical applicability of osteopontin as a new prognostic factor in patients with GC.

Acknowledgments The authors thank AIRC (Italian Association for Cancer Research) for financial support (IG 8738) of this nonprofit research. This report would not have been possible without the valuable contribution of the pathologists from the participating center. Moreover, the following investigators contributed to the study: Katia Fiorella Dotti, Arpine Gevorgyan, Francesca Dominioni, Elisa Sottotetti, Claudia Maggi , and Marta Caporale (Fondazione IRCCS Istituto Nazionale Tumori, Milan); Davide Poli (Clinical Research Laboratory, IRCCS Istituto di Ricerche Farmacologiche "Mario Negri," Milan), Francesco Di Costanzo (Azienda Ospedaliera-Università Careggi, Florence); Enrico Aitini (Azienda Ospedaliera "Carlo Poma," Mantova); Francesca Spada (Istituto Oncologico Europeo, Milan); Gerardo Rosati (Azienda Ospedaliera "San Carlo," Potenza); Donato Nitti (Azienda Ospedaliera di Padova, Padua); Francesca Pucci (Azienda Ospedaliera di Parma); Alberto Zaniboni (Istituto Ospedaliero Fondazione Poliambulanza, Brescia); Dino Amadori (IRST Forli); Tiziana Pressiani (Istituto Clinico Humanitas, Rozzano); Gianni Sanna (Istituto Ospedaliero dell'Università di Sassari); Maurizio Cantore (Ospedale Civico di Carrara); Barbara Venturini (Ospedale Infermi Rimini); Roberto Labianca (Ospedali Riuniti Bergamo); Paola Bolzoni (Presidio Ospedaliero "Serbelloni" di Gorgonzola-Melegnano); Carmine Pinto (Azienda Ospedaliera Sant'Orsola Malpighi Bologna); Corrado Boni (Arcispedale Santa Maria Nuova Azienda Ospedaliera di Reggio Emilia); Lorenza Landi (Presidio Ospedaliero di Livorno); Hector José Soto Parra (Policlinico Vittorio Emanuele Catania-Presidio Gaspare Rodolico); and Luigi Cavanna (Ospedale Civile "Guglielmo da Saliceto" Piacenza).

Conflict of interest The authors declare that they have no conflict of interest.

\section{References}

1. Parkin DM, Bray F, Ferlay J, Pisani P. Global cancer statistics, 2002. CA Cancer J Clin. 2005;55:74-108.

2. Paoletti X, Oba K, Burzykowski T, Michiels S, Ohashi Y, Pignon JP, et al. Benefit of adjuvant chemotherapy for resectable GC: a meta-analysis. JAMA. 2010;303:1729-37.

3. Macdonald JS, Smalley SR, Benedetti J, Hundahl SA, Estes NC, Stemmermann GN, et al. Chemoradiotherapy after surgery compared with surgery alone for adenocarcinoma of the stomach or gastroesophageal junction. N Engl J Med. 2001;345:725-30.

4. Cunningham D, Allum WH, Stenning SP, Thompson JN, Van de Velde CJ, Nicolson M, et al. Perioperative chemotherapy versus surgery alone for resectable gastroesophageal cancer. N Engl J Med. 2006;355:11-20.

5. Miceli R, Tomasello G, Bregni G, Di Bartolomeo M, Pietrantonio F. Adjuvant chemotherapy for gastric cancer: current evidence and future challenges. World J Gastroenterol. 2014;20:4516-25 review.

6. Shah MA, Khanin R, Tang L, Janjigian YY, Klimstra DS, Gerdes $\mathrm{H}$, et al. Molecular classification of gastric cancer: a new paradigm. Clin Cancer Res. 2011;17:2693-701.

7. Pietrantonio F, De Braud F, Da Prat V, Perrone F, Pierotti MA, Gariboldi M, et al. A review on biomarkers for prediction of treatment outcome in gastric cancer. Anticancer Res. 2013;33:1257-66.

8. Pignatelli M, Vessey CJ. Adhension molecules: novel molecular tools in tumor pathology. Hum Pathol. 1994;25:849-56.
9. Cao DX, Li ZJ, Jiang XO, Lum YL, Khin E, Lee NP, et al. Osteopontin as potential biomarker and therapeutic target in gastric and liver cancers. World J Gastroenterol. 2012;18:3923-30.

10. Song G, Ouyang G, Mao Y, Ming Y, Bao S, Hu T. Osteopontin promotes gastric cancer metastasis by augmenting cell survival and invasion through Akt-mediated HIF-1alpha up-regulation and MMP9 activation. J Cell Mol Med. 2009;13:1706-18.

11. Tang H, Wang J, Bai F, Zhai H, Gao J, Hong L, et al. Positive correlation of osteopontin, cyclooxygenase-2 and vascular endothelial growth factor in gastric cancer. Cancer Invest. 2008;26:60-7.

12. Bajetta E, Floriani I, Di Bartolomeo M, Labianca R, Falcone A, Di Costanzo F, et al. Randomized trial on adjuvant treatment with FOLFIRI followed by docetaxel and cisplatin versus 5-fluorouracil and folinic acid for radically resected gastric cancer. Ann Oncol. 2014;25:1373-8.

13. Oba K, Paoletti X, Alberts S, Bang YJ, Benedetti J, Bleiberg H, et al. Disease-free survival as a surrogate for overall survival in adjuvant trials of GC: a meta-analysis. J Natl Cancer Inst. 2013;105:1600-7.

14. Jung H, Lee HH, Song KY, Jeon HM, Park CH. Validation of the seventh edition of the American Joint Committee on Cancer TNM staging system for gastric cancer. Cancer. 2011;117:2371-8.

15. Ito T, Hashimoto $\mathrm{Y}$, Tanaka E, Kan $\mathrm{T}$, Tsunoda $\mathrm{S}$, Sato F, et al. An inducible short-hairpin RNA vector against osteopontin reduces metastatic potential of human esophageal squamous cell carcinoma in vitro and in vivo. Clin Cancer Res. 2006; 12:1308-16.

16. Jawhari A, Jordan S, Poole S, Browne P, Pignatelli M, Farthing MJ. Abnormal immunoreactivity of the E-cadherin-catenin complex in gastric carcinoma: relationship with patient survival. Gastroenterology. 1997;112:46-54.

17. Chau I, Norman AR, Cunningham D, Waters JS, Oates J, Ross PJ. Multivariate prognostic factor analysis in locally advanced and metastatic esophago-gastric cancer-pooled analysis from three multicenter, randomized, controlled trials using individual patient data. J Clin Oncol. 2004;22:2395-403.

18. Miceli R, Lusa L, Mariani L. Revising a prognostic index developed for classification purposes: an application to GC data. J Appl Stat. 2004;31:817-30.

19. Kattan MW, Karpeh MS, Mazumdar M, Brennan MF. Postoperative nomogram for disease-specific survival after an R0 resection for gastric carcinoma. J Clin Oncol. 2003;21:3647-50.

20. Dai N, Bao Q, Lu A, Li J. Protein expression of osteopontin in tumor tissues is an independent prognostic indicator in gastric cancer. Oncology. 2007;72:89-96.

21. Higashiyama M, Ito T, Tanaka E, Shimada Y. Prognostic significance of osteopontin expression in human gastric carcinoma. Ann Surg Oncol. 2007;14:3419-27.

22. Imano M, Satou T, Itoh T, Sakai K, Ishimaru E, Yasuda A, et al. Immunohistochemical expression of osteopontin in gastric cancer. J Gastrointest Surg. 2009;13:1577-82.

23. Wu CY, Wu MS, Chiang EP, Wu CC, Chen YJ, Chen CJ, et al. Elevated plasma osteopontin associated with gastric cancer development, invasion and survival. Gut. 2007;56:782-9.

24. Chen X, Meng T, Hao B, Zhang Z, Zhang G. Genetic polymorphisms in the osteopontin promoter increases the risk of distance metastasis and death in Chinese patients with gastric cancer. BMC Cancer. 2012;12:477.

25. Wang ZM, Cui YH, Li W, Chen SY, Liu TS. Lentiviral-mediated siRNA targeted against osteopontin suppresses the growth and metastasis of gastric cancer cells. Oncol Rep. 2011;25:997.

26. Buyse M, Sargent DJ, Grothey A, Matheson A, de Gramont A. Biomarkers and surrogate end points-the challenge of statistical validation. Nat Rev Clin Oncol. 2010;7:309-17. 